\title{
A Study of the Design of Wireless Medical Sensor Network based u- Healthcare System
}

\author{
Ronnie D. Caytiles and Sungwon Park ${ }^{1 *}$ \\ ${ }^{1}$ Hannam University \\ 133 Ojeong-dong, Daeduk-gu, Daejeon, Korea \\ rdcaytiles@gmail.com,sungwon@hnu.kr \\ *Correspondent Author: Sungwon Park*(sungwon@hnu.kr)
}

\begin{abstract}
Wireless Medical Sensor Networks and Radio Frequency Identification has been widely adopted in both medical and healthcare industry. This paper deals with the study of a WMSN and RFID based u-Healthcare system. An efficient framework for a u-Healthcare system utilizing RFID and WMSN has been presented. The system is capable of monitoring the patient's medical status by using RFID body sensor and wirelessly transmits the medical data to a local workstation (WMSN gateway) before transmitting it to the central database server. Due to the patient's movements, WMSN node's movements will be patterned with the functionality of the Mobile IPv6.
\end{abstract}

Keywords: MIPv6, WMSNs, RFID, u-Healthcare

\section{Introduction}

The adoption of Wireless Medical Sensor Networks (WMSNs) in the medical and healthcare industry has been widespread and very effective. In hospitals, patients can carry or even put on wearable sensors that can detect health-related parameters (e.g., glucose level, heart rate, etc.) and can monitor the patient continuously using wireless network. Sensors can be embedded or placed on human body (wearable), wirelessly connected and sharing information to monitor the patient's body conditions.

Hospitals and home-based healthcare services have been using the combination of both active and passive Radio Frequency Identification (RFID) technology. A radio-frequency identification system uses tags, or labels attached to the patient's body parts to be monitored. RFID readers send a signal to the tag and read its response. The RFID reader transmits an encoded radio signal to read the tag, and then the RFID tag receives the message and responds with its identification and other measured medical information [2]. Hospitals may use infrared monitors installed in medical facility rooms to collect data from transmissions of RFID badges and tags worn by patients and employees, as well as from tags assigned to medical facility assets, such as mobile medical devices.

An RFID tag may be incorporated with browser-based software to increase its effectiveness. This software allows hospital staff, nurses, and patients to see real-time data relevant to each unit of tracked equipment or personnel. Real-time data is stored and archived 
to make use of historical reporting functionality and to prove compliance with various industry regulations [2,4]. This combination of RFID real-time locating system hardware and software provides a powerful data collection tool for facilities seeking to improve operational efficiency and reduce costs.

This paper deals with the study of a WMSN and RFID based u-Healthcare system. An efficient framework for a u-Healthcare system utilizing RFID and WMSN has been presented. The system is capable of monitoring the patient's medical status by using RFID body sensor and wirelessly transmits the medical data to a local workstation (WMSN gateway) before transmitting it to the central database server. Proper administration of medical services is carried out in the local workstation based on the queries with the central database which contains all the information for the patient. Patients can be alerted in case of emergency through their wearable device and can also receive messages with their Smartphones. The medical staff on the workstation will also receive an alert message indicating that the patient status needs attention. A medical service depending on the status of the patient will be prescribed or applied to the patient. Due to the patient's movements, WMSN node's movements will be patterned with the functionality of the Mobile IPv6.

The rest of this paper is organized as follows: Section 2 discusses some related technologies; Section 3 outlines the utilization of WMSNs and RFID to u-Healthcare system; and the concluding remarks in Section 4.

\section{Related Technologies}

\subsection{Wireless Medical Sensor Networks}

A wireless medical sensor network (WMSNs) refers to spatially distributed autonomous devices using sensors to monitor physical or environmental conditions (such as temperature, sound, pressure, etc.) that incorporates a gateway that provides wireless connectivity to remotely distributed nodes $[1,5]$. WMSNs utilize wireless protocols depending on the application requirements that include available standards such as $2.4 \mathrm{GHz}$ radios based on either IEEE 802.15.4 or IEEE 802.11 (Wi-Fi) standards or proprietary radios, which are usually $900 \mathrm{MHz}$.

WMSN applications include health care, utilities, and remote monitoring. In health care, wireless devices make patient monitoring and healthcare easy and more effective. Wireless networks applicable for u-healthcare systems can be classified into three groups:

- In-body networks are used for communication between sensors implanted in body and a receiver outside body. Examples of which are implantable pacemaker and ICD, and smart capsule that are used to transfer bio-information, which can measure in the body, to an external device.

- On-body networks are used for communication between sensors attached on body and data gathering devices. Examples of which are bio-shirt, wrist watches, and ring sensors attached on body to transfer sensing data to a local processing.

- External Networks are used in home and mobile healthcare that communicates between sensors, devices or a location processing unit on personal can communicate with a remote server. 
Table 1. Wireless Networks for u-Health

\begin{tabular}{|c|c|}
\hline $\begin{array}{c}\text { Classification of a Wireless Network in } u- \\
\text { Healthcare }\end{array}$ & Wireless Network Technology \\
\hline In-Body & $\begin{array}{c}\text { Low-frequency inductive coupling, ISM, } \\
\text { MICS }\end{array}$ \\
\hline On-Body (Wearable) & $\begin{array}{c}\text { WMTS, RFID, Bluetooth, Zigbee, WLAN } \\
\text { (Wi-fi) }\end{array}$ \\
\hline External Network & $\begin{array}{c}\text { Cellular Networks } \\
\text { (CDMA/HSDPA/GPRS/EDGE/UMTS), } \\
\text { Wibro, Satellite }\end{array}$ \\
\hline
\end{tabular}

Compared to wired networks, wireless networks seem advantageous as a communication network for u-Health. Wireless network can be used between each layer of u-Health service model. Sensors which measure health condition in sensing layer transfer sensing data to a gateway node or directly to an external server. Then the gateway node delivers the data to a central server with mobile network.

\subsection{RFID}

A Radio-frequency identification (RFID) refers to the wireless non-contact use of radiofrequency electromagnetic fields to transfer data, for the purposes of automatically identifying and tracking tags attached to objects [2]. The tags contain electronically stored information powered by and read at short ranges (a few meters) via magnetic fields (electromagnetic induction) and may be embedded in the tracked object.

RFID tags can be embedded and can be attached to clothing, possessions, or even implanted within people. Many medical and healthcare industries have been effectively used the adoption of RFID. They can easily monitor and collect data from transmissions of RFID badges and tags worn by patients and employees, as well as from tags assigned to facility assets, such as mobile medical devices. A physical RFID tag may be incorporated with browser-based software to increase its efficacy. This software allows for different groups or specific hospital staff, nurses, and patients to see real-time data relevant to each piece of tracked equipment or personnel. Real-time data is stored and archived to make use of historical reporting functionality and to prove compliance with various industry regulations. This combination of RFID real-time locating system hardware and software provides a powerful data collection tool for facilities seeking to improve operational efficiency and reduce costs.

\subsection{Mobile IPv6}

Mobile IP is a standard that allows users with mobile devices whose IP addresses are associated with one network to stay connected when moving to a network with a different IP address. When a user leaves the network with which his device is associated (home network) and enters the domain of a foreign network, the foreign network uses the Mobile IP protocol to inform the home network of a care-of address to which all packets for the user's device should be sent [3].

The mobile IP protocol allows location-independent routing of IP datagrams on the Internet. Each mobile node is identified by its home address disregarding its current location in. While away from its home network, a mobile node is associated with a care-of address which identifies its current location and its home address is associated with the local endpoint of a 
tunnel to its home agent. Mobile IP specifies how a mobile node registers with its home agent and how the home agent routes datagrams to the mobile node through the tunnel [3].

Mobile IP is most often found in wireless WAN environments where users need to carry their mobile devices across multiple LANs with different IP addresses.

Mobile IPv6 is a version of Mobile IP - a network layer IP standard used by electronic devices to exchange data across a packet switched internetwork. Mobile IPv6 allows an IPv6 node to be mobile- to arbitrarily change its location on an IPv6 network- and still maintain existing connections [3]. IPv6 (Internet Protocol version 6) is the successor to the well known IPv4 protocol, commonly known as IP.

\section{Wireless Sensor Network Based u-Healthcare}

The system architecture for a u-Healthcare system utilizing RFID and WMSN is capable of monitoring the patient's medical status by using RFID body sensor and wirelessly transmits the medical data to a local workstation (WMSN gateway) before transmitting it to the central database server as shown in Figure 1. The patient put on an RFID wearable sensor to continuously monitor and capture multiple physiological signals such as body temperature, blood pressure, ECG, respiration rate, and etc. The medical information will be transmitted via an RFID reader and will be recorded temporarily on the nearest workstation before it will be transmitted to the central database server wherein the complete patient's medical records are stored and updated regularly. Doctors or medical professionals then can analyze and check the patient's status, through the monitoring system accessible within the medical/healthcare facility or remotely using their Smartphones or PDAs equipped with the medical monitoring system application.

The u-healthcare system performs such important functions and applications outlined as follows:

(1) An individual's personal information with his current health data is collected with RFID tags worn by patients. Various bio-signals are checked through RFID tags as blood sugar, blood pressure, pulse, active mass, weight, muscle quantity, body fat quantity, body fat rate, basal metabolic rate, body mass index, etc.

(2) The health data that are collected through RFID tags are amplified and transmitted through RFID readers and sent to the local workstation.

(3) The transmitted health data are stored and updated in the central database server.

(4) (5) The patient's health information are then interpreted and analyzed based on the medical relevant facts. The system real-time monitors the individual's health status based on his/her personalized health plans and it subsequently renders medicallyrelevant advice to maintain a healthy lifestyle, remotely.

- The system also provides reminders for scheduled therapy and appointments and can electronically schedule medical appointments.

- The system also alerts healthcare agencies whenever an emergency situation is detected. It should provide monitoring services for depression, hypertension, diabetes and cardio-vascular related diseases. Staffs who are monitoring biosignal at the healthcare center call emergency service in case abnormal symptom appears and then visit patients and check their state directly. 


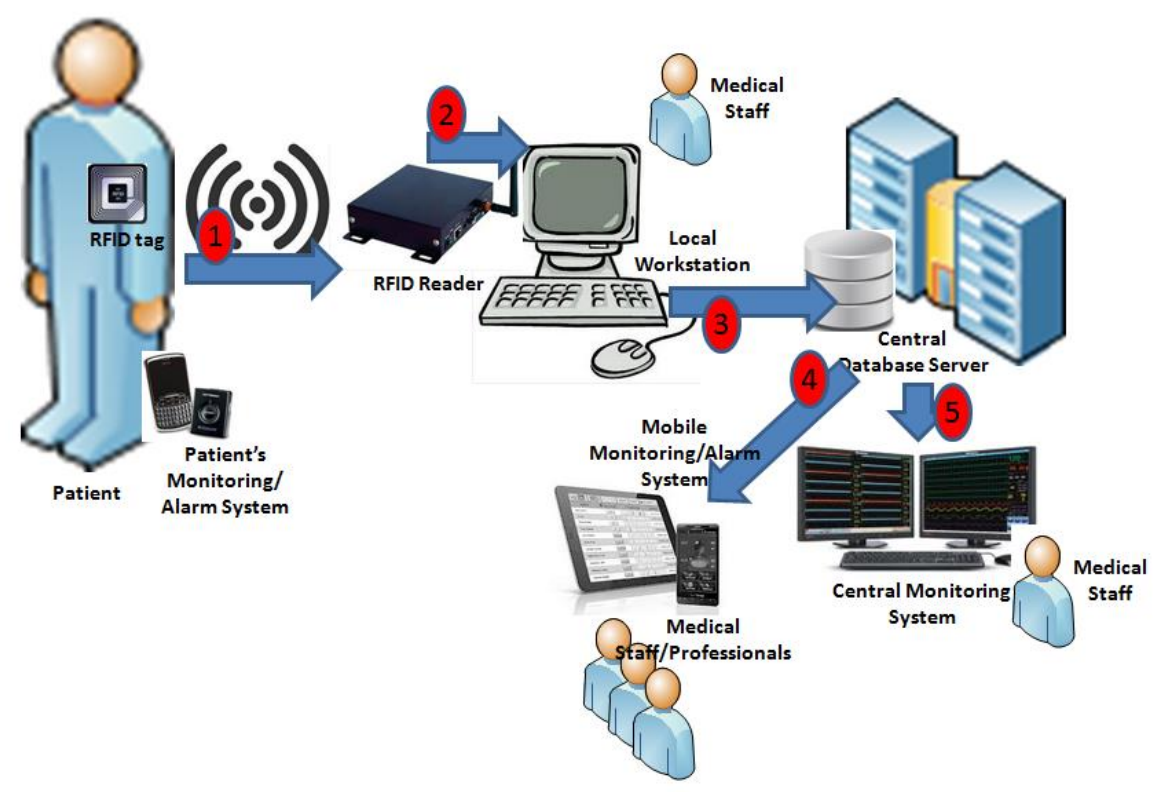

Figure 1. The Design for u-Healthcare System Utilizing WMSN and RFID

Proper administration of medical services can also be carried out in the local workstation based on the queries with the central database which contains all the information for the patient. Patients can be alerted in case of emergency through their wearable device and can also receive messages with their Smartphones. The medical staff on the workstation as well as those medical professionals monitoring the system will also receive an alert message indicating that the patient status needs attention. A medical service depending on the status of the patient will be prescribed or applied to the patient. Due to the patient's movements, WMSN node's movements will be patterned with the functionality of the Mobile IPv6 as shown in Figure 2.

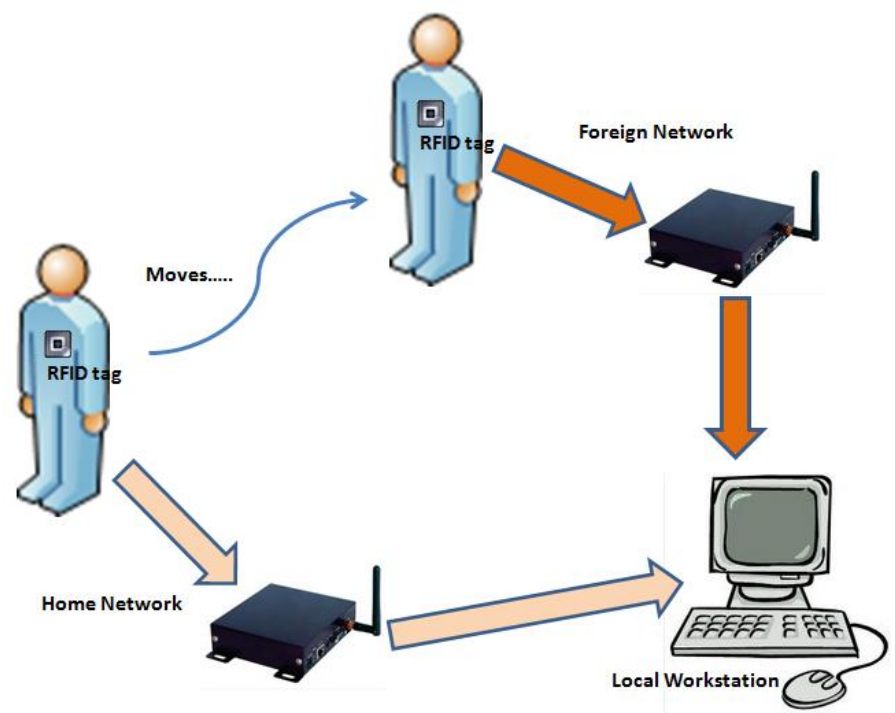

Figure 2. Movement of Wireless Sensor Nodes 
As the patient move from one location to another, RFID tags that are connected to the patient will be read by nearest RFID reader installed within the medical/healthcare facility. The process of connectivity and transmission will be the same as the connectivity of the moving nodes in MIPv6.

\section{Conclusion and Future Works}

This paper has presented an efficient framework for u-Healthcare system utilizing RFID and WMSN. The system is capable of monitoring the patient's medical status by using RFID body sensor and wirelessly transmits the medical data to a local workstation (WMSN gateway) before transmitting it to the central database server. Proper administration of medical services is carried out in the local workstation based on the queries with the central database which contains all the information for the patient. Patients can be alerted in case of emergency through their wearable device and can also receive messages with their Smartphones. The medical staff on the workstation will also receive an alert message indicating that the patient status needs attention. A medical service depending on the status of the patient will be prescribed or applied to the patient. Due to the patient's movements, WMSN node's movements will be patterned with the functionality of the Mobile IPv6.

\section{Acknowledgments}

This paper has been supported by the 2014 Hannam University Research Fund.

\section{References}

[1] National Instruments, "What Is a Wireless Sensor Network?”, (2012) May 5, http://www.ni.com/whitepaper/7142/en/.

[2] http://en.wikipedia.org/wiki/Radio-frequency_identification.

[3] D. Johnson, C. Perkins, et al., "Mobility Support in IPv6", IETF RFC 3775, (2004) June.

[4] H. Alemdar and C. Ersoy, "Wireless sensor networks for healthcare: A survey", Computer Networks, Elsevier, vol. 54, (2010), pp. 2688-2710.

[5] J. G. Ko, C. Lu, M. B. Srivastava, J. A. Stankovic, A. Terzis and M. Welsh, "Wireless Sensor Networks for Healthcare", Proceedings of the IEEE, DOI: 10.1109/JPROC.2010.2065210, vol. 98, no. 11, (2010) November, pp. 1947-1960.

\section{Authors}

Ronnie D. Caytiles Currently, he is a graduate student on the Integrated Course in College of Engineering from Hannam University. His research interests include ubiquitous e-health care, Multimedia Healthcare, and sensor network.

Sungwon Park received the B.S., M.S. and Ph.D. degrees in Nursing from Yonsei University in 1998, 2002 and 2005 respectively. From 2006 to February 28, 2010, she was an assistant professor in the college of nursing at Hyechon University. Since March 1, 2010, he has been a professor in the Department of Nursing at Hannam University, Daejeon, Korea. 